\title{
An Animal Model for the Treatment of Alzheimer's Disease: Potential for a New Direction in Therapies that Enhance the Epigenic Mechanisms of Memory Formation
}

\author{
Howard B Eichenbaum*, \\ 'Center for Memory and Brain, Boston University, Boston, MA, USA
}

Neuropsychopharmacology (20 I0) 35, 853-854; doi: I 0.I 038/npp.2009.205

\begin{abstract}
Alzheimer's disease $(\mathrm{AD})$ is a neurogenerative disorder of which the etiology has been related to the development of beta amyloid plaques and neurofibrillary tangles. Currently there are no therapies to prevent these abnormalities, or otherwise halt the progression of this devastating disease that robs its victims progressively of their memory and their identity, and ultimately hastens death. At this time there are also few symptomatic treatments, and those that are available ameliorate cognitive loss primarily in the early stage, albeit with significant side effects.
\end{abstract}

Animal models are useful for the identification of new drug targets for amyloid-associated neurodegeneration, because we now have transgenic mice that overexpress amyloid protein and because behavioral assays for mice have been developed to measure the impairment in episodic memory that occurs in the earliest stage of AD. Furthermore, as described by Kilgore et al, in the current issue of Neuropsychopharmacology, mouse models that have been used to explore the fundamental cellular and molecular bases of normal memory might also be exploited in translational directions to manipulate those mechanisms in generating new drug therapies for memory disorders.

Kilgore et al (2010) examined mice that overexpress amyloid precursor protein (APP) and a variant of presenilin (PS1), resulting in the aggressive aggregation of amyloid plaques that characterize familial, early-onset $\mathrm{AD}$. The choice of drug target was based on earlier identification of an important role of histone acetyltransferases that catalyze acetylation of histone proteins, which relax chromatin structure and facilitate the transcription of genes involved in memory formation. Specifically, the authors examined the role of these epigenic events by exploring the effects of drugs that inhibit histone deacetylase (HDAC), which

\footnotetext{
*Correspondence: Dr HB Eichenbaum, Department of Psychology, Center for Memory and Brain, Boston University, Boston, MA 02115 , USA, Tel: + | 617353 |426, Fax: + | 617353 |414,

E-mail: hbe@bu.edu
}

normally brings transcription to a halt by removing the histone acetyl groups. The authors postulated that HDAC inhibitors should extend gene transcription and consequently improve memory, particularly under conditions in which the capacity for memory is compromised.

First, the authors determined the age of onset of the memory disorder in APP/PS1 mice. Four-month-old APP/ PS1 mice exhibit a normal ability in learning to fear an environmental context in which they have received mild footshocks, and they, as well as wild-type (WT) mice, maintain this memory overnight. However, at 6 months of age, severe memory impairment emerges in APP/PS1 mice at the 1-day test. Importantly, 6-month-old APP/PS1 mice have normal levels of motor activity before shock, exhibit a normal immediate response to shock, and can normally maintain the fear memory for an hour. This pattern of behavioral findings indicates that APP/PS1 mice provide a valid model of the selective long-term memory disorder that is characteristic of the early stage of AD.

Then the authors chronically treated 6-month-old WT and APP/PS1 mice with one of three HDAC inhibitors: sodium valproate, sodium butyrate or vorinostat (SAHA; Zolinza) and evaluated their memory at 1 day after training. HDAC inhibitors did not affect motor activity or the immediate freezing response to shock; nor did these drugs affect the memory performance in WT mice. However, each of the drugs fully eliminated the memory deficit observed in untreated APP/PS1 mice at the 1-day retention test. Furthermore, continued treatment actually prevented a normal decline in memory strength that occurs in WT mice after an additional 2 weeks; thus, APP/PS1 mice exhibited above-normal preservation of memory in a long-term retention test. These findings provide compelling evidence that the three drugs, which have in common the inhibition of a class of HDAC isoforms, all selectively restore memory capacity in a mouse model of early-onset AD.

It is important to acknowledge that HDAC inhibitors do not directly affect the cause of memory decline, but instead appear to act by improving the suboptimal memory 
performance, which is a consequence of amyloid aggregation. Notably, histone acetylation is not affected in APP/PS1 mice, although histone acetylation is elevated by the drug treatments. HDAC inhibitors did not improve the memory in WT mice, but the authors cite other evidence suggesting that, under weak training conditions in which performance is not optimized, HDAC inhibitors do improve memory in normal young animals. Thus, when memory is already optimal, further driving of the system is not effective; but when the system is compromised, improvements are obtained. Combining all these considerations, although the range of functions affected by HDC inhibitors is poorly understood, it seems most likely that HDAC inhibitors work by optimizing the molecular cascade that supports memory, countering the challenge to memory that results from amyloid accumulation.

There is much more work to do in moving towards a clinical trial. The authors recommend caution in overinterpreting a memory enhancement that may be dependent on age, genetic background and training parameters.
Nonetheless, the current results raise optimism for a new direction in drug development that seeks to address memory impairments not by attacking the cause of the disorder but instead by boosting the sluggish molecular machinery of a memory system that is compromised by any underlying etiology. Ultimately, though, a final cautionary note is that this strategy is not directed at a cure, and is unlikely to dramatically affect the unrelenting course of neurodegeneration in $\mathrm{AD}$.

\section{DISCLOSURE}

The author declares no conflict of interest.

\section{REFERENCES}

Kilgore M, Miller CA, Fass DM, Hennig KM, Haggarty SJ, Sweatt JD et al. (2010). Inhibitors of class 1 histone deacetylases reverse contextual memory deficits in a mouse model of Alzheimer's disease. Neuropsychopharmacology 35: 870-880. 